\title{
The intake of a high-fat diet and grape seed procyanidins induces gene expression changes in peripheral blood mononuclear cells of hamsters: capturing alterations in lipid and cholesterol metabolisms
}

\author{
Antoni Caimari $\cdot$ Anna Crescenti $\cdot$ Francesc Puiggròs • \\ Noemí Boqué $\cdot$ Lluís Arola $\cdot$ Josep Maria del Bas
}

Received: 24 July 2014/ Accepted: 23 October 2014/Published online: 13 November 2014

(c) Springer-Verlag Berlin Heidelberg 2014

\begin{abstract}
We previously demonstrated that hamsters that were fed either a standard diet (STD) or a high-fat diet (HFD) and treated with a grape seed procyanidin extract (GSPE) showed decreased adiposity and circulating levels of free fatty acids compared with hamsters treated with a vehicle (Caimari et al. in Int J Obes 37:576-83, 2013, doi:10.1038/ijo.2012.75). Here, we tested whether the gene expression changes in peripheral blood mononuclear cells (PBMCs) can reflect these metabolic effects and the dyslipidaemia produced by the HFD feeding in the same cohort of animals. The mRNA levels of a subset of genes were also studied in the liver in order to evaluate the capacity of PBMCs to reflect the metabolic adaptations that occur in this organ. In PBMCs, we reported a simultaneous up-regulation of the lipid-related genes involved in both the anabolic (ppary, accl and gpat) and the catabolic (ppara, $u c p 2$, atgl and $h s l$ ) pathways in response to the GSPE treatment, similar but no identical to previous observations
\end{abstract}

Electronic supplementary material The online version of this article (doi:10.1007/s12263-014-0438-y) contains supplementary material, which is available to authorized users.

A. Caimari $(\bowtie)$ · A. Crescenti · F. Puiggròs · N. Boqué ·

J. M. del Bas

Grup de Recerca en Nutrició i Salut (GRNS). Centre Tecnològic

de Nutrició i Salut (CTNS), TECNIO, CEICS, Avinguda

Universitat, 1, 43204 Reus, Spain

e-mail: antoni.caimari@ctns.cat

L. Arola

Centre Tecnològic de Nutrició i Salut (CTNS), TECNIO,

CEICS, Avinguda Universitat, 1, 43204 Reus, Spain

L. Arola

Nutrigenomics Research Group, Departament de Bioquímica i Biotecnologia, Universitat Rovira i Virgili, Marcel•lí Domingo s/n, 43007 Tarragona, Spain in retroperitoneal white adipose tissues of these animals. Furthermore, the key cholesterol metabolism genes srebp2 and $l d l r$ were significantly down-regulated in PBMCs of both HFD-fed groups compared with the STD groups. Although the expression of srebp2 in the liver followed a similar pattern to that obtained in PBMCs, no comparable changes were found between the liver and PBMCs in the expression of most of the studied genes. In conclusion, our results highlight the potential of PBMCs as a high accessible tissue for the indirect study of cholesterol and adipose tissue metabolism dynamics.

Keywords Procyanidins - PBMCs - Dyslipidaemia · Adipose tissue $\cdot$ Liver - Lipid and cholesterol metabolism . Nutritional biomarkers

\section{Introduction}

Peripheral blood mononuclear cells (PBMCs), which include lymphocytes and monocytes, circulate throughout the body and are thus more easily obtained than other samples, such as adipose tissue, liver or muscle. The use of these cells in nutrition-related studies is increasing due to the number of different studies performed in both animals and humans that have demonstrated the relationship of PBMCs-captured metabolic changes with the fasting and feeding cycles (Bouwens et al. 2007; Caimari et al. 2010a, b; Oliver et al. 2013) and with the intake of specific nutrients or bioactive food compounds such as fatty acids, proteins, carbohydrates and polyphenols (van Erk et al. 2006; Crujeiras et al. 2008; Bouwens et al. 2009; Konstantinidou et al. 2009; Khymenets et al. 2009; Bouwens et al. 2010; Camargo et al. 2010; Rudkowska et al. 2011; Van Dijk et al. 2012a, b; Tomé-Carneiro et al. 2013; van der Velpen et al. 2013; 
Afman et al. 2014). Furthermore, it has been shown that PBMCs can act as biomarkers of the metabolic adaptations that occur in different tissues such as adipose tissue, liver and muscle (Caimari et al. 2010a; Rudkowska et al. 2011; Oliver et al. 2013; O'Grada et al. 2013; Konieczna et al. 2014), although there are some controversial results about this issue (Rudkowska et al. 2011; O'Grada et al. 2013; Afman et al. 2014). In addition, other studies have demonstrated that PBMCs can reflect gene expression signatures that are characteristic of different diseases linked to a sedentary lifestyle and increased food intake, such as atherosclerosis (Kang et al. 2006), chronic heart failure (Cappuzzello et al. 2009) and metabolic syndrome (D'Amore et al. 2013).

Polyphenols are bioactive food compounds that are primarily present in fruits and vegetables and exert many protective effects against cardiovascular disease (Bladé et al. 2010; Quiñones et al. 2013). In this context, our group and others have reported many healthy beneficial effects of a grape seed procyanidin extract (GSPE) (rich in monomers and oligomers of flavan-3-ols, the most abundant polyphenols in the human diet), on different pathologies clustered within the metabolic syndrome, such as insulin resistance, dyslipidaemia, obesity, hypertension and inflammation (Del Bas et al. 2005; Pinent et al. 2006; Quesada et al. 2009; Bladé et al. 2010; Terra et al. 2011; Belcaro et al. 2013; Caimari et al. 2013). Interestingly, some studies have shown that polyphenols, which may be isolated or included in extracts or foods, such as olive oil and cocoa, are also able to regulate the gene expression profiles of PBMCs (Konstantinidou et al. 2009; Khymenets et al. 2009; Camargo et al. 2010; Crescenti et al. 2013; Tomé-Carneiro et al. 2013; van der Velpen et al. 2013). However, to the best of our knowledge, only one study has reported gene expression changes of PBMCs after the consumption of a grape seed extract rich in polyphenols in combination with resveratrol in hypertensive men with type 2 diabetes mellitus (Tomé-Carneiro et al. 2013).

The Golden Syrian hamster is an experimental animal model that shares features of its cholesterol metabolism with humans; therefore, the results obtained potentially allow a better extrapolation to humans compared with those results obtained in rats and mice (Zhang et al. 2009). This feature is of special interest because the disturbance of the cholesterol and lipoprotein metabolism is linked to the appearance of cardiovascular disease, a very important cause of morbidity and mortality worldwide (Roy 2014). Considering the potential of PBMCs to reflect both the nutritional status and the different common diet-related diseases, the analysis of the gene expression levels in PBMCs of hamsters can emerge as a promising strategy to detect new potential biomarkers of different metabolic disturbances clustered with cardiovascular disease, such as dyslipidaemia, and to elucidate the impact of consuming different foods' bioactive compounds on the amelioration or prevention of these pathologies.

In a previous study, we demonstrated that the administration of GSPE ( $25 \mathrm{mg} / \mathrm{kg}$ of body weight) to hamsters fed either a standard diet (STD) or a high-fat diet (HFD) significantly reduced the adiposity and circulating levels of free fatty acids (NEFAs) in the animals fed both the STD and the HFD and partially reversed the mild dyslipidaemia induced by the HFD feeding (Caimari et al. 2013).

The aim of the present study was to investigate, in the same cohort of animals, whether the metabolic alterations related to lipid metabolism that were produced by consuming a HFD and the healthy beneficial effects observed as a consequence of GSPE intake were reflected at the mRNA level in PBMCs. For this purpose, the mRNA expression of a set of key genes involved in cholesterol and lipid metabolism was analysed by Q-PCR in PBMCs. In addition, the regulation of some of these genes in response to the HFD and the GSPE treatment was also analysed in the liver of these hamsters in order to evaluate the ability of PBMCs to reflect the metabolic adaptations that can occur in this organ.

\section{Methods and materials}

\section{Procyanidin extract}

GSPE was kindly provided by Les Dérives Résiniques et Terpéniques (Dax, France), and its composition was previously described by Arola et al. (Arola-Arnal et al. 2013). Briefly, this extract contained procyanidin dimer $(33.1 \%)$, catechin $(20.8 \%)$, epicatechin gallate $(12.7 \%)$, epicatechin $(12.6 \%)$, dimer gallate $(9.1 \%)$, procyanidin trimer $(6.5 \%)$ and gallic acid $(4.1 \%)$.

\section{Animals}

The animal protocol followed in this study was reviewed and approved by the Bioethical Committee of the University Rovira i Virgili (Tarragona, Spain), and guidelines for the use and care of laboratory animals of the University were followed.

The animals used in the present experiment have been previously studied (Caimari et al. 2013). Briefly, 3-monthold male Golden Syrian hamsters (Charles River Laboratories, Barcelona, Spain) weighing $130 \mathrm{~g}$ were housed singly at $22{ }^{\circ} \mathrm{C}$ with a light/dark period of $12 \mathrm{~h}$ (lights on at 09:00) and with free access to food and water. The hamsters were randomly distributed into two experimental groups $(n=16)$ and fed ad libitum with either a STD or a HFD for 15 days. Then, the animals were divided into four 
groups $(n=7-8)$, depending on the treatment received. Every day, two groups were orally treated with a syringe containing $25 \mathrm{mg}$ of GSPE per $\mathrm{kg}$ body weight dissolved in low-fat condensed milk (the STD-GSPE and HFD-GSPE groups). The other two groups received the same volume of low-fat condensed milk as the vehicle (the STD and HFD groups). The STD (3.9 kcal $/ \mathrm{g})$ contained $10 \%$ calories from fat, whereas the HFD $(4.1 \mathrm{kcal} / \mathrm{g})$ contained $21 \%$ calories from fat (primarily lard) and $0.9 \mathrm{~g} / \mathrm{kg}$ cholesterol. The diets were prepared in pelleted form by Research Diet Services BV (Wijk bij Duurstede, The Netherlands).

On day 15 of the GSPE/vehicle treatment, the hamsters were fasted for $5 \mathrm{~h}$ (from 09:00 to 14:00) and anaesthetised with sodium pentobarbital, and blood was collected through cardiac puncture. Blood samples $(2-2.5 \mathrm{~mL})$ were diluted (1:1) with phosphate-buffered saline ( $\mathrm{pH} 7.4)$, and PBMCs were isolated by Ficoll gradient separation, according to the instructions indicated by the manufacturer (GE Healthcare Bio Sciences, Barcelona, Spain). The PBMCs pellet was carefully resuspended with $1.5 \mathrm{~mL}$ of erythrocyte lysis buffer $\left(155 \mathrm{mM} \mathrm{NH}_{4} \mathrm{Cl}, 10 \mathrm{mM} \mathrm{KHCO}_{3}\right.$ and $100 \mu \mathrm{M}$ EDTA, pH 7.4), incubated at room temperature for $5 \mathrm{~min}$ and centrifuged at $400 \mathrm{~g}$ for $10 \mathrm{~min}$. Finally, the supernatant was aspirated, and the PBMCs pellet was stored at $-70{ }^{\circ} \mathrm{C}$ until RNA analysis. Plasma was obtained by centrifugation, and the liver and different white adipose tissue depots (retroperitoneal-RWAT, mesentericMWAT, epididymal-EWAT and inguinal-IWAT) were rapidly removed after death, weighed, frozen in liquid nitrogen and stored at $-70{ }^{\circ} \mathrm{C}$ until further analysis.

\section{Adiposity index}

The adiposity was determined by an adiposity index computed for each hamster as the sum of the EWAT, IWAT, MWAT and RWAT depot weights and expressed as a percentage of the total body weight.

\section{RNA extraction}

PBMCs and liver total RNA were extracted using Tripure reagent (Roche Diagnostic Barcelona, Spain) and purified with Qiagen RNeasy Mini Kit spin columns (Izasa, Barcelona, Spain) according to the manufacturer's instructions. RNA yield was quantified in a NanoDrop ND-1000 spectrophotometer (NanoDrop Technologies Wilmintog, Delaware, USA), and the integrity of the RNA was confirmed using agarose gel electrophoresis.

Gene expression analysis

$0.5 \mu \mathrm{g}$ of PBMCs and liver total RNA was denatured and then reverse transcribed to cDNA using MuLV reverse transcriptase (according to Applied Biosystem's procedure) in a Perkin Elmer 9,700 Thermal Cycler (Norwalk, CT). For Q-PCR, the Light Cycler 480 II System with the SYBR Green I Master Mix (Roche Diagnostic Barcelona, Spain) was used. The PCR mixture for the different assays contained $2 \mu \mathrm{L}$ of sample cDNA (diluted $1 / 5$ or $1 / 10$ ), $0.55 \mu \mathrm{m}$ of each primer, $6 \mu \mathrm{L}$ of Light Cycler 480 SYBR Green and PCR-grade sterile water to a final volume of $11 \mu \mathrm{L}$. Thermocycling conditions were as follows: $95{ }^{\circ} \mathrm{C}$ for 5 min for initial denaturation and activation of Taq polymerase, followed by 45 thermal cycles of $95{ }^{\circ} \mathrm{C}$ for $10 \mathrm{~s}$, $60{ }^{\circ} \mathrm{C}$ for $10 \mathrm{~s}$ and $72{ }^{\circ} \mathrm{C}$ for $10 \mathrm{~s}$, with a ramping rate of 4.4, 2.2 and $4.4{ }^{\circ} \mathrm{C} / \mathrm{s}$, respectively. Fluorescence was measured once during each $72{ }^{\circ} \mathrm{C}$ step. Following the amplification process, a melting curve analysis was performed by heating the plate at $95{ }^{\circ} \mathrm{C}$ for $5 \mathrm{~s}$, incubating at $65^{\circ} \mathrm{C}$ for $1 \mathrm{~min}$ followed by an slowly heating $\left(0.11^{\circ} \mathrm{C} / \mathrm{s}\right)$ to $97{ }^{\circ} \mathrm{C}$ (with five acquisitions per ${ }^{\circ} \mathrm{C}$ ). Fluorescence was monitored continuously during the melting experiment. Crossing point values ( $\mathrm{Cp}$ ) were determined using the Light Cycler software. The primers for the different genes are described in supplementary Table 1 and were obtained from Biomers.net (Ulm, Germany). The relative expression of each mRNA was calculated as a percentage of the STD group, using the $2^{-\Delta \Delta C_{t}}$ method (Livak and Schmittgen 2001) with $\beta$-actin as the reference gene.

\section{Statistical analysis}

Data are expressed as the mean \pm SEM. The differences between groups were analysed using a two-way ANOVA to evaluate the effects of diet (STD or HFD) and GSPE (or + ) and the interaction between these factors (diet $\mathrm{x}$ GSPE). When one or both of the main effects were statistically significant, a one-way ANOVA followed by the least significance difference (LSD) test was used to determine the mean differences between treatments. Independent Student's t tests for comparisons across groups were applied when necessary. Linear relationships between key variables were tested using Pearson's correlation coefficients. Analyses were performed using the statistical software SPSS Statistics 18 (SPSS, Inc., Chicago, IL, USA). Statistical significance was considered at $p<0.05$.

\section{Results}

Tissue weights and plasma parameters

Data on the tissue weights and plasma parameters of the experimental model used in this study have been previously published (Caimari et al. 2013). Briefly, compared 

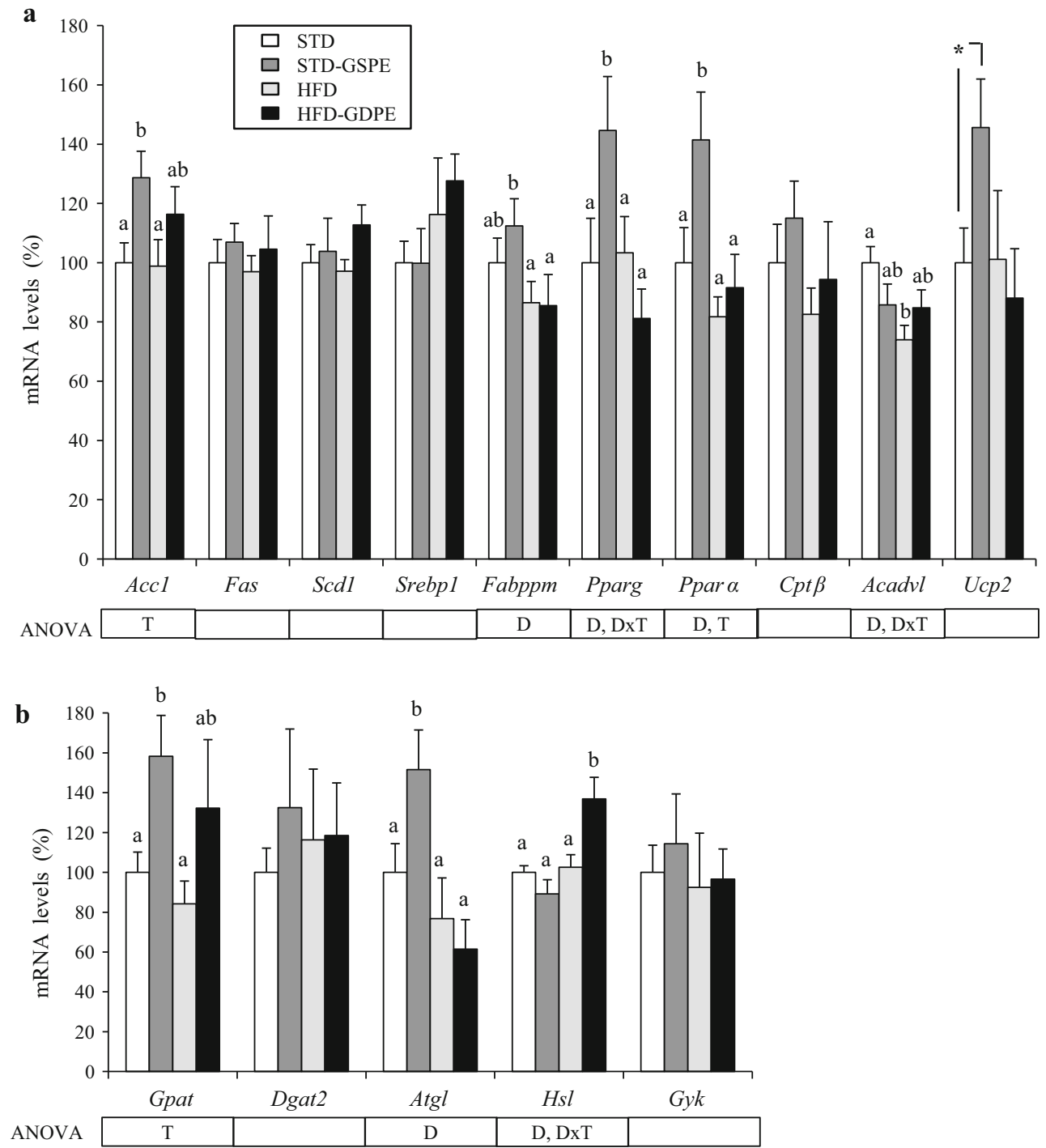

Fig. 1 The mRNA expression levels of genes related to fatty acid metabolism (a) and glycerolipid synthesis and lipolysis (b) in PBMCs of hamsters fed with a STD or a high-fat diet for 30 days receiving a daily oral dose of GSPE (25 mg per kg body weight) (the STD-GSPE and HFD-GSPE groups) or the vehicle (the STD and HFD groups) for the last 15 days. The gene expression results represent the mean \pm SEM $(n=7-8)$ of the ratios of specific mRNA levels to $\beta$-actin, expressed as a percentage versus the STD group which was set to $100 \%$. $D$ the effect of the type of diet, $T$ the effect of GSPE treatment, DxT, the interaction of the type of diet and GSPE treatment (two-way ANOVA, $p<0.05$ ). ${ }^{a b}$ Mean values with unlike letters were significantly different among groups (one-way ANOVA and LSD post

with the STD group, the HFD group showed increased circulating levels of triglycerides ( $85 \%$ higher), total cholesterol (28\% higher) and phospholipids (17\% higher) and tended to have higher RWAT and liver weights than did the STD group. The GSPE treatment significantly decreased the weight of the different white adipose depots (the RWAT, MWAT, EWAT and IWAT) and, consequently, the adiposity indices in both of the hoc test, $p<0.05$ ). *Versus the STD group (Student's $t$ test, $p<0.05)$. Acadvl, acyl-coa dehydrogenase, very long chain; Acc, acetyl-coenzyme a carboxylase; Atgl, adipose triglyceride lipase; Cpt1 $\beta$, carnitine palmitoyltransferase beta; Dgat2, diacylglycerol acyltransferase-2; Fabppm: fatty acid-binding protein plasma membrane; Fas, fatty acid synthase; Gyk: glycerol kinase; Gpat, glycerol3-phosphate acyltransferase; Hsl, hormone-sensitive lipase; Lpl: lipoprotein lipase; Ppara, peroxisome proliferator-activated receptor alpha; Ppar $\gamma$, peroxisome proliferator-activated receptor gamma; Scd1, stearoyl-coa desaturase-1; Srebp1, sterol regulatory elementbinding protein $1 ; U c p 2$, uncoupling protein 2 (mitochondrial, proton carrier)

GSPE-treated groups. This effect was more evident in the HFD-GSPE group than in the STD-GSPE group. Furthermore, both GSPE-treated groups showed lower circulating levels of NEFAs compared with their respective non-treated groups. GSPE treatment also reversed the increase in phospholipids induced by the HFD feeding but failed to reduce the circulating levels of cholesterol and triglycerides. 


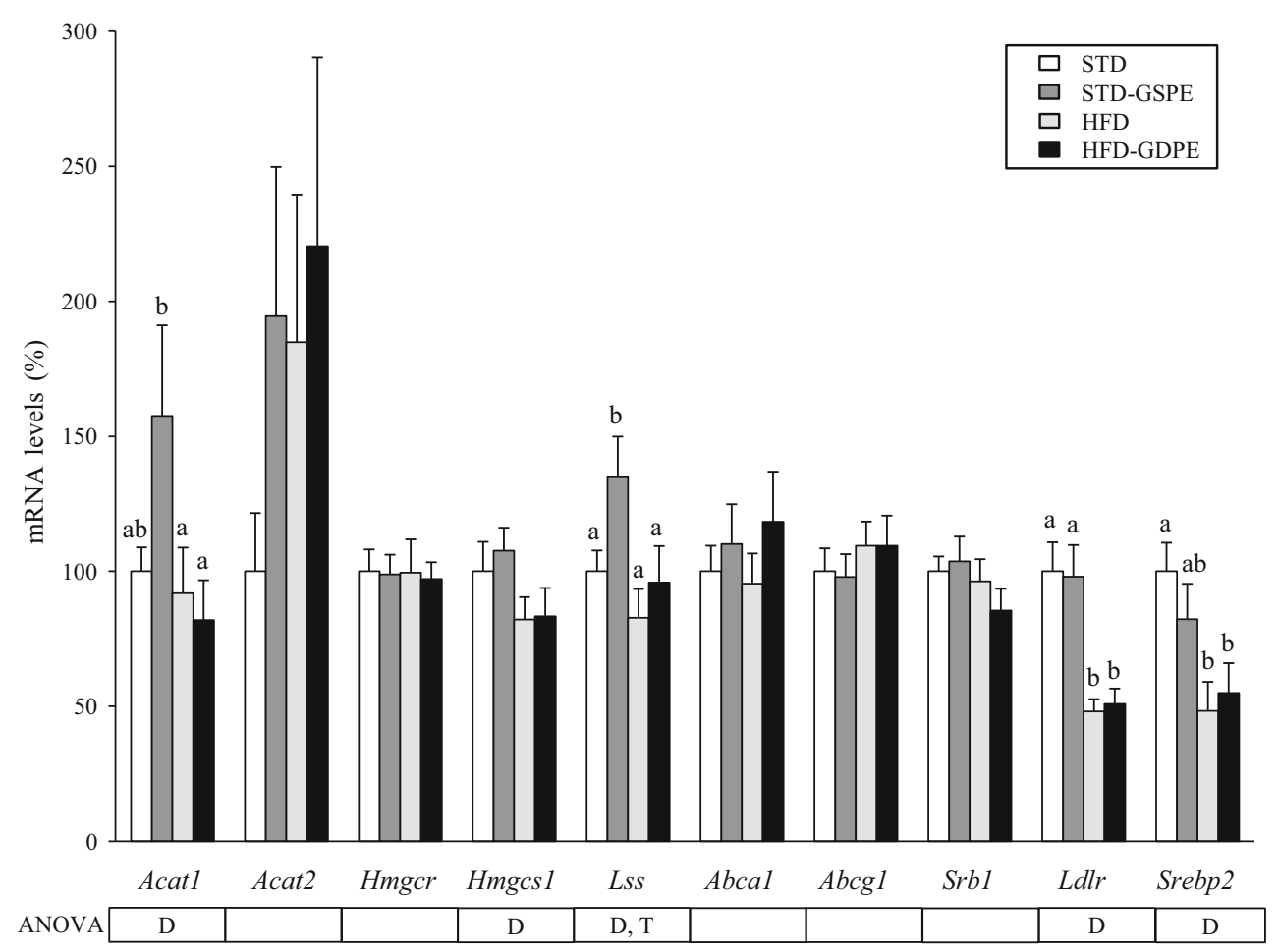

Fig. 2 The mRNA expression levels of genes related to cholesterol homoeostasis in PBMCs. Hamsters were fed with a STD or a high-fat diet for 30 days and received a daily oral dose of GSPE (25 mg per $\mathrm{kg}$ body weight) (the STD-GSPE and HFD-GSPE groups) or vehicle (the STD and HFD groups) for the last 15 days. The gene expression results represent the mean $\pm \operatorname{SEM}(n=7-8)$ of the ratios of specific mRNA levels to $\beta$-actin, expressed as a percentage versus the STD group which was set to $100 \%$. $D$ the effect of the type of diet; $T$ the effect of GSPE treatment (two-way ANOVA, $p<0.05$ ). ${ }^{\text {ab }}$ Mean values with unlike letters were significantly different among groups

\section{Gene expression in PBMCs}

The mRNA levels of a set of genes related to fatty acid metabolism, glycerolipid synthesis, lipolysis and cholesterol homoeostasis were analysed in PBMCs.

The gene expression of two key enzymes involved in lipid anabolism, ACC1 and GPAT was induced in response to GSPE treatment in both the STD and the HFD animals, with this effect being more evident in the STD-GSPE group than in the HFD-GSPE group (Fig. 1a, b). In addition, the GSPE treatment increased the mRNA levels of atgl, ppar $\alpha$, ppar $\gamma$ and ucp2 in the STD-GSPE animals but failed to up-regulate these genes in the HFD-GSPE group (Fig. 1a, b). On the contrary, the gene expression of the key lipolytic enzyme HSL was increased only in the HFDGSPE animals in response to GSPE administration (Fig. 1b).

With regard to the genes involved in cholesterol metabolism, the HFD intake significantly decreased the mRNA levels of acatl, hmgcsl, lss, ldlr and srebp 2 in both the HFD and the HFD-GSPE groups (Fig. 2). This effect (one-way ANOVA and LSD post hoc test, $p<0.05$ ). Abcal, ATPbinding cassette, sub-family A (ABC1), member 1; Abcg1, ATPbinding cassette, sub-family G (WHITE), member 1; Acat1, Acat1 acetyl-CoA acetyltransferase 1; Acat2, Acat2 acetyl-CoA acetyltransferase 2; Hmgcr, 3-hydroxy-3-methylglutaryl-CoA reductase; Hmgcs 1, 3-hydroxy-3-methylglutaryl-CoA synthase 1 (soluble); Lss, lanosterol synthase (2,3-oxidosqualene-lanosterol cyclase); $L d l r$, lowdensity lipoprotein receptor; $S r b 1$, scavenger receptor class B, member 1; Srebp2, sterol regulatory element-binding protein 2

was more evident for the $l d l r$ and srebp 2 genes, in which the drop in the expression approached $50 \%$ in both HFDfed groups (Fig. 2). No significant changes in response to the HFD feeding were found either in the expression of acat 2 or in the expression of key genes involved in the cholesterol efflux from macrophages to HDL (abcal, $a b c g 1$ and $s r b 1$ ) (Fig. 2). The GSPE treatment significantly increased the mRNA levels of $l s s$ in the STD-GSPE group, and a similar trend was observed for the acatl gene, although the differences did not reach statistical significance (Fig. 2).

\section{Gene expression in the liver}

To evaluate whether the changes in response to the HFD intake and the GSPE treatment observed in PBMCs reflect the metabolic adaptations that occur in the liver, we checked the expression of selected genes involved in both cholesterol homoeostasis (abcgl, acat2, hmgcr, ldlr, srbl and srebp2) and lipid metabolism (atgl, cptla, dgat2, fas, gpat, ppara and srebpl) in this organ. 

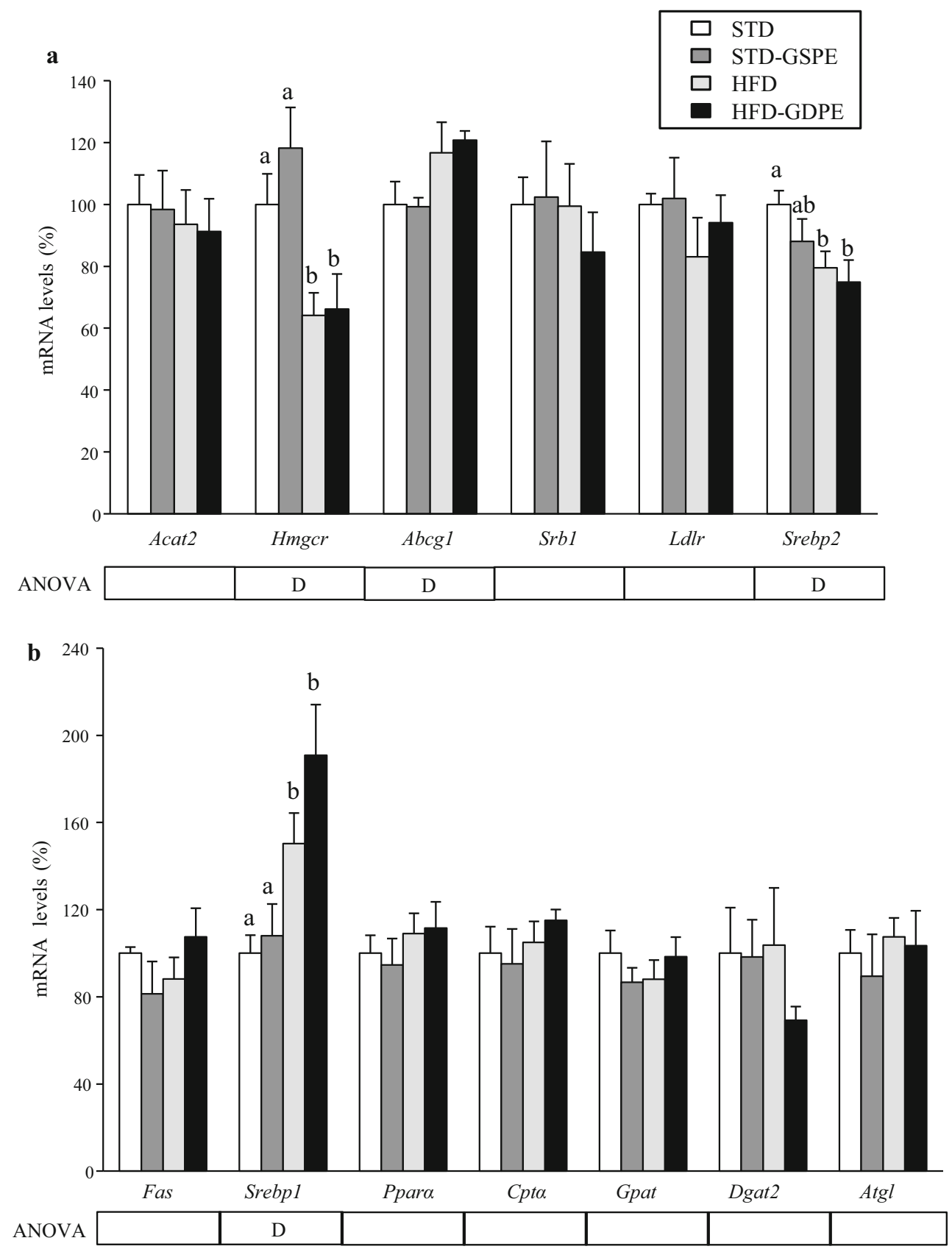

Fig. 3 The mRNA expression levels of genes related to cholesterol (a) and lipid metabolism (b) in the liver of hamsters fed with a STD or a high-fat diet for 30 days receiving a daily oral dose of GSPE $(25 \mathrm{mg}$ per kg body weight) (the STD-GSPE and HFD-GSPE groups) or the vehicle (the STD and HFD groups) for the last 15 days. The gene expression results represent the mean $\pm \operatorname{SEM}(n=7-8)$ of the ratios of specific mRNA levels to $\beta$-actin, expressed as a percentage versus the STD group which was set to $100 \%$. D the effect of the type of diet (two-way ANOVA, $p<0.05$ ). ${ }^{\text {ab }}$ Mean values with unlike letters were significantly different among groups (one-way ANOVA and

Concerning the cholesterol metabolism, the HFD intake decreased the mRNA levels of srebp2 in the liver of both HFD groups, similar to that occurred in PBMCs, although the percentage of decrease in this organ was only about
LSD post hoc test, $p<0.05)$. Abcg1, ATP-binding cassette, subfamily G (WHITE), member 1; Acat2, Acat2 acetyl-CoA acetyltransferase 2; Atgl, adipose triglyceride lipase; Cpt $1 \alpha$, carnitine palmitoyltransferase alpha; Dgat2, diacylglycerol acyltransferase-2; Fas, fatty acid synthase; Gpat, glycerol-3-phosphate acyltransferase; Hmgcr, 3-hydroxy-3-methylglutaryl-CoA reductase; Ldlr, low-density lipoprotein receptor; Ppard, peroxisome proliferator-activated receptor alpha; Srb1, scavenger receptor class B, member 1; Srebp1, sterol regulatory element-binding protein $1 ;$ Srebp 2 , sterol regulatory element-binding protein 2

$20 \%$ (Fig. 3a). The HFD feeding also slightly increased the expression of $a b c g l$ in the liver of both the HFD and the HFD-GSPE groups (Fig. 3a). In addition, and different to that was observed in PBMCs, a marked down-regulation 
of the hmgcr mRNA levels was reported in the liver of both groups of animals that were challenged with the HFD, whereas no significant changes in the mRNA levels of $l d l r$ were observed in the liver of these two groups of animals when compared with their respective controls (Fig. 3a).

The HFD feeding produced a significant increase in the mRNA levels of srebpl in both the HFD and the HFDGSPE animals (Fig. 3b). This pattern of expression was also observed in PBMCs although the differences between groups did not reach statistical significance (Fig. 1a). Neither the GSPE treatment nor the HFD feeding affected the expression of the other genes involved in lipid metabolism in the liver (Fig. 3b).

Correlations of the mRNA levels in PBMCs with tissue weights and with RWAT and liver mRNA levels

Strong negative correlations between the mRNA levels of $h s l$ in PBMCs and the adiposity index $(r=-0.662$, $p=0.007)$ and the RWAT weight $(r=-0.669$, $p=0.006$ ) were found only when the HFD-fed groups were considered. Furthermore, in these groups, the IWAT weight was also correlated negatively with the $h s l$ gene expression values $(r=-0.541, p=0.037)$.

No significant correlations were found between the mRNA levels of any of the genes analysed either in PBMCs and the RWAT or in PBMCs and the liver. However, it is worth noting that the mRNA levels of $h s l$ in PBMCs tended to positively correlate with those obtained in the RWAT when all of the animals were considered together $(r=0.346, p=0.066)$ and when only the HFD groups were selected ( $r=0.492, p=0.074)$.

\section{Discussion}

In a previous study, we found that the intake of a HFD, the treatment with GSPE and the combination of both nutritional interventions affects the lipid metabolism of the adipose tissue (Caimari et al. 2013). We proposed the activation of both the $\beta$-oxidation and the glycerolipid/free fatty acid (GL/FFA) cycles at the mRNA level in the RWAT as putative mechanisms by which GSPE, when orally administered to hamsters fed either a STD or a HFD, reduced the weight of different white adipose tissue depots and decreased the circulating levels of NEFAs (Caimari et al. 2013). In the present work, we have analysed the changes at the gene expression level in PBMCs of the same cohort of hamsters in order to assess whether these cells can be a source of biomarkers able to reflect changes of the adipose status. Interestingly, and similar to the observations in the RWAT, we found a simultaneous increase in the mRNA levels of the lipid-related genes involved in both the catabolic (ppard, ucp2, atgl and hsl) and the anabolic (ppar $\gamma$, accl and gpat) pathways in the PBMCs of GSPE-treated animals, suggesting that the GL/ FFA cycle could also be enhanced in these cells in response to GSPE treatment. In addition, in the present study, the expression patterns of $h s l$ and gpat found in PBMCs were very similar to those observed previously in RWAT (Caimari et al. 2013). These results are in agreement with those obtained in rats by Caimari et al. (2010a), which also found a similar expression pattern of lipidrelated genes, such as cptla, fas, ppary and srebpl, between PBMCs and the mesenteric white adipose tissue in response to fasting and feeding fluctuations. Furthermore, although no significant correlations between the mRNA levels of $h s l$ and gpat in both tissues were obtained, robust negative correlations between the mRNA levels of $h s l$ in PBMCs and RWAT weights and adiposity indices were found in the HFD-fed groups. Though we do not know whether there is a mechanistic or cause-effect link among $h s l$ expression in PBMCs and the physiological status of the adipose tissue, the expression of this gene in PBMCs could be considered as an interesting biomarker candidate. Further research is needed in order to assess its applicability in humans and for the study of altered states related with the adipose tissue status such as obesity, insulin resistance or metabolic syndrome. The upregulation of the ppar $\alpha$ and atgl observed in PBMCs in response to GSPE was only evident in animals that were challenged with STD, contrary to the RWAT observations for the same animals (Caimari et al. 2013). A very similar pattern of expression was observed in PBMCs for the ucp2 and ppary genes. As a possible explanation, the response to GSPE in the HFD-fed animals could be masked by the higher levels of circulating factors (triglycerides and cholesterol) as a consequence of the HFD intake because this could trigger a lower response of these animals' PBMCs to the metabolic signals that are activated as a consequence of GSPE treatment. In this sense, a higher nutritional response of different lipid- and cholesterol-related genes, such as ppar $\gamma$, cptla, srebp2 and lss, in the PBMCs of healthy rats compared with cafeteria-fed obese rats was previously described by Caimari et al. (2010a, b) and Oliver et al. (2013). However, it is important to consider that the same HFD-fed animals showed a higher response to GSPE in the RWAT than did the STD-GSPE group in terms of weight loss and gene expression activation (Caimari et al. 2013). Therefore, a different sensitivity to GSPE between adipocytes and PBMCs cannot be ruled out. These results suggest that PBMCs do not completely reflect the elements of lipid metabolism in the adipose tissue, in agreement with the results obtained by O'Grada et al. (2013), who showed no correlations in the expressions of lipid- and cholesterol- 
related genes, such as PPAR $P P A R \gamma$ and $A B C G 1$, between PBMCs and the subcutaneous adipose tissue of subjects submitted to oral lipid and glucose tolerance tests.

Hamsters have been proposed as a very interesting preclinical model for the study of cholesterol metabolism due to the features that these animals share with humans but not with rats or mice (Zhang et al. 2009). Our results in PBMCs suggest that these cells are sensitive to the plasma cholesterol levels. Thus, hmgcsl, a gene that codes for a key enzyme involved in the synthesis of mevalonate from acetyl-CoA during cholesterol synthesis, and $l s s$, which plays a role in lanosterol synthesis (Horton 2002; Espenshade and Hughes 2007) were slightly down-regulated in PBMCs of both HFD and HFD-GSPE animals. In addition, the gene coding for the LDL receptor, which mediates the cellular uptake of cholesterol from cholesterol-rich LDL, chylomicron remnants and VLDL remnants (IDL) (Daniels et al. 2009), was also down-regulated (50\% decrease) in the PBMCs of both groups of dyslipidaemic hamsters. Most interestingly, the mRNA levels of the gene coding for the master regulator of cholesterol homoeostasis, SREBP2 (Horton 2002; Espenshade and Hughes 2007; BengoecheaAlonso and Ericsson 2007), were decreased in the PBMCs of the two groups of hamsters that developed dyslipidaemia, compared with their respective healthy controls. This effect was even more evident in the HFD group that was not treated with GSPE. It is to highlight that hmgcoas, lss and $l d l r$ are known to be regulated by SREBP2 (Horton 2002) and that all of these genes present the same expression pattern as that observed for srebp 2 . The cellular levels of cholesterol are tightly regulated at transcriptional level through the activation or inhibition of genes that are involved in cholesterol synthesis and uptake via SREBP2 (Horton 2002; Espenshade and Hughes 2007; BengoecheaAlonso and Ericsson 2007). Therefore, when cells are depleted of sterols, there is an up-regulation of the genes required for the synthesis and uptake of cholesterol via the activation of SREBP2. However, in sterol-overloaded cells, there is an inactivation of SREBP2 with the consequent repression of these genes to return the cholesterol levels to normal (Horton 2002; Espenshade and Hughes 2007; Bengoechea-Alonso and Ericsson 2007). In this scenario, it is tempting to speculate that in our study, the intake of a HFD, which contains nearly $0.1 \%$ cholesterol, inhibits the expression of genes involved in cholesterol synthesis and uptake in PBMCs to counteract the high supply of this compound to the cells, thereby maintaining sterol homoeostasis. Interestingly, the common expression pattern between SREBP2 and the various genes involved in cholesterol synthesis observed in the present study was previously shown by Caimari et al. (2010b) in the PBMCs of rats submitted to fasting/re-feeding fluctuations. Altogether, these results suggest that SREBP2 governs cholesterol synthesis in PBMCs, tightly regulating the responses of these cells to different nutritional challenges.

Interestingly, our analyses in the liver showed that the expression pattern of srebp 2 was similar to that observed in PBMCs, suggesting similar modulatory mechanisms in cholesterol metabolism in both tissues. However, no significant changes in the expression of the gene that codifies for the LDL receptor were found in the liver of both HFDfed groups, and the most important change at the mRNA level was found in the expression of hmgcr, the gene that codes for HMG-CoA reductase, the rate-limiting enzyme in cholesterol synthesis (Horton 2002; Espenshade and Hughes 2007), suggesting different sensitivity to the HFD between genes expressed in both tissues. Therefore, our results suggest that, in hamsters, although the gene expression profile in PBMCs reflect the metabolic adaptations related to cholesterol metabolism that occur as a consequence of a HFD intake, it does not accurately reflect elements of cholesterol metabolism in the liver.

Different studies have shown a modulation of the expression of cholesterol metabolism-related genes, such as abcal, abcgl and $s r b l$, in macrophages responding to treatment with polyphenols quercetin, resveratrol and extra virgin olive oil, another source of polyphenols (Voloshyna et al. 2013; Lee et al. 2013; Helal et al. 2013). However, in our study, of all the analysed genes that are involved in cholesterol homoeostasis, only lss was up-regulated in PBMCs in response to the administration of GSPE in the STD-GSPE animals. This discrepancy may depend on the use of extracts or purified molecules, differences among the polyphenols used or the cells in which the polyphenols have been tested (PBMCs or macrophages). The fact that the in vivo available data related to the effects of polyphenols on PBMCs gene expression do not report changes at the mRNA levels of the genes tested in the present study (Afman et al. 2014), together with the lack of response to GSPE observed in the liver of these animals, suggests that these genes are not very sensitive to the effects of these bioactive food compounds.

One limitation of the present study is the fact that it is difficult to discern whether the reported changes in PBMCs gene expression in response to GSPE intake are a consequence of the metabolic improvements derived from the chronic GSPE consumption, are directly induced by the intake of the extract or are provoked by the combination of both effects. Although some studies have demonstrated the ability of polyphenols administrated in one single dose to induce gene expression changes in PBMCs (Konstantinidou et al. 2009; Camargo et al. 2010), further acute studies analysing the short-term effects of GSPE on PBMCs gene expression could contribute to shed light on this issue. In addition, new studies focused on the effects of purified molecules obtained from GSPE (i.e. procyanidin dimer and 
trimers, catechin, epicatechin and epicatechin gallate) on PBMCs gene expression would make possible the elucidation of the main bioactive compounds that are responsible of the observed effects, which could be an starting point to obtain new valuable information about the effects of grape seed polyphenols on PBMCs from a mechanistic point of view.

In conclusion, we here report that the expression profiles of srebp 2 and $l d l r$ in PBMCs respond to changes in the metabolism of cholesterol induced by a high-fat diet. Though more research is still needed, our results suggest that these cells might be a valuable source of biomarkers in studies were different mechanisms affecting the metabolism of cholesterol need to be monitored with a minimum intervention or without tissue harvesting, including human studies. Taking into account that the GSPE treatment exerted profound effects in the lipid metabolism of the adipose tissue at the gene expression and physiological level, the changes observed in PBMCs suggest that these cells are able to capture different metabolic changes that take place in the adipose tissue. On the contrary, no link was found with the lipid metabolism in the liver, suggesting that PBMCs might be a useful source of biomarkers to study different events taking place in the adipose but not in the liver. Moreover, it is worth considering that these results have been found in hamsters, which share many common features of lipid metabolism with humans but not with rats or mice. Altogether, our results place PBMCs as a potential tool for the indirect study of the adipose tissue metabolism dynamics by means of a highly available tissue such as blood. Although more research is still needed, application to human studies seems feasible and valuable.

Acknowledgments The research leading to these results received funding from ACC1Ó (TECRD12-1-0005) and from the European Union's Seventh Framework Programme FP7 2007-2013 under grant agreement $\mathrm{n}^{\circ} 244995$ (BIOCLAIMS Project). We gratefully acknowledge the aid of Vanessa Grifoll and Silvia Pijuan, the laboratory technicians.

Conflict of interest Antoni Caimari, Anna Crescenti, Francesc Puiggròs, Noemí Boqué, Lluís Arola and Josep Maria del Bas declare that they have no conflict of interest.

Ethical standard All institutional and national guidelines for the care and use of laboratory animals were followed.

\section{References}

Afman L, Milenkovic D, Roche HM (2014) Nutritional aspects of metabolic inflammation in relation to health-insights from transcriptomic biomarkers in PBMC of fatty acids and polyphenols. Mol Nutr Food Res 1-13. doi:10.1002/mnfr.201300559

Arola-Arnal A, Oms-Oliu G, Crescenti A et al (2013) Distribution of grape seed flavanols and their metabolites in pregnant rats and their fetuses. Mol Nutr Food Res 57:1741-1752. doi:10.1002/ mnfr.201300032

Belcaro G, Ledda A, Hu S et al (2013) Grape seed procyanidins in pre- and mild hypertension: a registry study. Evid Based Complement Altern Med 313142. doi:10.1155/2013/313142

Bengoechea-Alonso MT, Ericsson J (2007) SREBP in signal transduction: cholesterol metabolism and beyond. Curr Opin Cell Biol 19:215-222. doi:10.1016/j.ceb.2007.02.004

Bladé C, Arola L, Salvadó M-J (2010) Hypolipidemic effects of proanthocyanidins and their underlying biochemical and molecular mechanisms. Mol Nutr Food Res 54:37-59. doi:10.1002/ mnfr.200900476

Bouwens M, Afman LA, Müller M (2007) Fasting induces changes in peripheral blood mononuclear cell gene expression profiles related to increases in fatty acid beta-oxidation: functional role of peroxisome proliferator activated receptor alpha in human peripheral blood mononuclear cells. Am J Clin Nutr 86:1515-1523

Bouwens M, Van De Rest O, Dellschaft N et al (2009) Fish-oil supplementation induces antiinflammatory gene expression profiles in human blood mononuclear cells. Am J Clin Nutr 90:415-424. doi:10.3945/ajen.2009.27680

Bouwens M, Bromhaar MG, Jansen J et al (2010) Postprandial dietary lipid-specific effects on human peripheral blood mononuclear cell gene expression profiles. Am J Clin Nutr 91:208-217. doi:10.3945/ajen.2009.28586

Caimari A, Oliver P, Keijer J, Palou A (2010a) Peripheral blood mononuclear cells as a model to study the response of energy homeostasis-related genes to acute changes in feeding conditions. OMICS 14:129-141. doi:10.1089/omi.2009.0092

Caimari A, Oliver P, Rodenburg W et al (2010b) Feeding conditions control the expression of genes involved in sterol metabolism in peripheral blood mononuclear cells of normoweight and dietinduced (cafeteria) obese rats. J Nutr Biochem 21:1127-1133. doi:10.1016/j.jnutbio.2009.10.001

Caimari A, del Bas JM, Crescenti A, Arola L (2013) Low doses of grape seed procyanidins reduce adiposity and improve the plasma lipid profile in hamsters. Int J Obes 37:576-583. doi:10. 1038/ijo.2012.75

Camargo A, Ruano J, Fernandez JM et al (2010) Gene expression changes in mononuclear cells in patients with metabolic syndrome after acute intake of phenol-rich virgin olive oil. BMC Genom 11:253. doi:10.1186/1471-2164-11-253

Cappuzzello C, Napolitano M, Arcelli D et al (2009) Gene expression profiles in peripheral blood mononuclear cells of chronic heart failure patients. 233-240. doi:10.1152/physiolgenomics.90364. 2008

Crescenti A, Solà R, Valls RM et al (2013) Cocoa consumption alters the global DNA methylation of peripheral leukocytes in humans with cardiovascular disease risk factors: a randomized controlled trial. PLoS One 8:e65744. doi:10.1371/journal.pone.0065744

Crujeiras AB, Parra D, Milagro FI et al (2008) Differential expression of oxidative stress and inflammation related genes in peripheral blood mononuclear cells in response to a low-calorie diet: a nutrigenomics study. OMICS 12:251-261. doi:10.1089/omi. 2008.0001

D'Amore S, Vacca M, Graziano G et al (2013) Nuclear receptors expression chart in peripheral blood mononuclear cells identifies patients with metabolic syndrome. Biochim Biophys Acta 1832:2289-2301. doi:10.1016/j.bbadis.2013.09.006

Daniels TF, Killinger KM, Michal JJ et al (2009) Lipoproteins, cholesterol homeostasis and cardiac health. Int $\mathrm{J}$ Biol Sci 5:474-488

Del Bas JM, Fernández-Larrea J, Blay M et al (2005) Grape seed procyanidins improve atherosclerotic risk index and induce liver CYP7A1 and SHP expression in healthy rats. FASEB J Off Publ Fed Am Soc Exp Biol 19:479-481. doi:10.1096/fj.04-3095fje 
Espenshade PJ, Hughes AL (2007) Regulation of sterol synthesis in eukaryotes. Annu Rev Genet 41:401-427. doi:10.1146/annurev. genet.41.110306.130315

Helal O, Berrougui H, Loued S, Khalil A (2013) Extra-virgin olive oil consumption improves the capacity of HDL to mediate cholesterol efflux and increases ABCA1 and ABCG1 expression in human macrophages. Br J Nut 109:1844-1855. doi:10.1017/ S0007114512003856

Horton JD (2002) Sterol regulatory element-binding proteins: transcriptional activators of SREBP structure and activation SREBP isoform function. Biochem Soc Trans 30:1091-1095

Kang J-G, Patino WD, Matoba S, Hwang PM (2006) Genomic analysis of circulating cells: a window into atherosclerosis. Trends Cardiovasc Med 16:163-168. doi:10.1016/j.tcm.2006.03. 006

Khymenets O, Fitó M, Covas M-I et al (2009) Mononuclear cell transcriptome response after sustained virgin olive oil consumption in humans: an exploratory nutrigenomics study. OMICS 13:7-19. doi:10.1089/omi.2008.0079

Konieczna J, Sánchez J, van Schothorst EM et al (2014) Identification of early transcriptome-based biomarkers related to lipid metabolism in peripheral blood mononuclear cells of rats nutritionally programmed for improved metabolic health. Genes Nutr 9:366. doi:10.1007/s12263-013-0366-2

Konstantinidou V, Khymenets O, Fito M et al (2009) Characterization of human gene expression changes after olive oil ingestion: an exploratory approach. Folia Biol 55:85-91

Lee S-M, Moon J, Cho Y et al (2013) Quercetin up-regulates expressions of peroxisome proliferator-activated receptor $\gamma$, liver $\mathrm{X}$ receptor $\alpha$, and ATP binding cassette transporter A1 genes and increases cholesterol efflux in human macrophage cell line. Nutr Re (New York, NY) 33:136-43. doi:10.1016/j.nutres.2012.11. 010

Livak KJ, Schmittgen TD (2001) Analysis of relative gene expression data using real-time quantitative PCR and the 2(-Delta Delta C(T)) Method. Methods (San Diego, Calif) 25:402-8. doi:10. 1006/meth.2001.1262

O'Grada CM, Morine MJ, Morris C et al (2013) PBMCs reflect the immune component of the WAT transcriptome-Implications as biomarkers of metabolic health in the postprandial state. Mol Nutr Food Res 1-13. doi:10.1002/mnfr.201300182

Oliver P, Reynés B, Caimari A, Palou A (2013) Peripheral blood mononuclear cells: a potential source of homeostatic imbalance markers associated with obesity development. Pflüg Archiv Eur J Physiol 465:459-468. doi:10.1007/s00424-013-1246-8

Pinent M, Bladé C, Salvadó MJ et al (2006) Procyanidin effects on adipocyte-related pathologies. Crit Rev Food Sci Nutr 46:543-550. doi:10.1080/10408390500354537

Quesada H, del Bas JM, Pajuelo D et al (2009) Grape seed proanthocyanidins correct dyslipidemia associated with a highfat diet in rats and repress genes controlling lipogenesis and
VLDL assembling in liver. Int J Obes 33:1007-1012. doi:10. 1038/ijo.2009.136

Quiñones M, Miguel M, Aleixandre A (2013) Beneficial effects of polyphenols on cardiovascular disease. Pharmacol Res Off J the Ital Pharmacol Soc 68:125-131. doi:10.1016/j.phrs.2012.10.018

Roy S (2014) Atherosclerotic Cardiovascular Disease Risk and Evidence-based Management of Cholesterol. N Am J Med Sci 6:191-198. doi:10.4103/1947-2714.132916

Rudkowska I, Raymond C, Ponton A et al (2011) Validation of the use of peripheral blood mononuclear cells as surrogate model for skeletal muscle tissue in nutrigenomic studies. OMICS 15:1-7. doi:10.1089/omi.2010.0073

Terra X, Pallarés V, Ardèvol A et al (2011) Modulatory effect of grape-seed procyanidins on local and systemic inflammation in diet-induced obesity rats. J Nutr Biochem 22:380-387. doi:10. 1016/j.jnutbio.2010.03.006

Tomé-Carneiro J, Larrosa M, Yáñez-Gascón MJ et al (2013) Oneyear supplementation with a grape extract containing resveratrol modulates inflammatory-related microRNAs and cytokines expression in peripheral blood mononuclear cells of type 2 diabetes and hypertensive patients with coronary artery disease. Pharmacol Res Off J Ital Pharmacol Soc 72:69-82. doi:10.1016/ j.phrs.2013.03.011

Van der Velpen V, Geelen A, Schouten EG et al (2013) Estrogen receptor-mediated effects of isoflavone supplementation were not observed in whole-genome gene expression profiles of peripheral blood mononuclear cells in postmenopausal, equolproducing women. J Nutr 143:774-780. doi:10.3945/jn.113. 174037

Van Dijk SJ, Mensink M, Esser D et al (2012a) Responses to high-fat challenges varying in fat type in subjects with different metabolic risk phenotypes: a randomized trial. PLoS One 7:e41388. doi:10.1371/journal.pone.0041388

Van Dijk SJ, Feskens EJM, Bos MB et al (2012b) Consumption of a high monounsaturated fat diet reduces oxidative phosphorylation gene expression in peripheral blood mononuclear cells of abdominally overweight men. 1219-1225. doi:10.3945/jn.111. 155283

Van Erk MJ, Blom WAM, van Ommen B, Hendriks HFJ (2006) High-protein and high-carbohydrate breakfasts differentially change the transcriptome of human blood cells. Am J Clin Nutr 84:1233-1241

Voloshyna I, Hai O, Littlefield MJ et al (2013) Resveratrol mediates anti-atherogenic effects on cholesterol flux in human macrophages and endothelium via PPAR $\gamma$ and adenosine. Eur $\mathbf{J}$ Pharmacol 698:299-309. doi:10.1016/j.ejphar.2012.08.024

Zhang Z, Wang H, Jiao R et al (2009) Choosing hamsters but not rats as a model for studying plasma cholesterol-lowering activity of functional foods. Mol Nutr Food Res 53:921-930. doi:10.1002/ mnfr.200800517 